\title{
Experimental overview of collective flow with identified particles at RHIC and the LHC
}

\author{
Panos Christakoglou ${ }^{1, a}$ \\ ${ }^{1}$ Nikhef, Science Park 105, 1098XG Amsterdam, The Netherlands
}

\begin{abstract}
Anisotropic flow studies play a crucial role in improving our understanding of the behaviour and the nature of matter created in collisions of heavy ions. In particular, the study of elliptic flow $\left(v_{2}\right)$ for identified particles can be used to constrain the initial conditions and the value of shear viscosity over entropy density ratio. It also allows to determine the role of the hadronic rescattering phase in the development of flow. In these proceedings I review the results from measurements of $v_{2}$ for identified particles from the RHIC and LHC heavy-ion physics programs.
\end{abstract}

\section{Introduction}

One of the most remarkable predictions of the Standard Model of particle physics is that, at sufficiently high densities and temperatures, the protons and neutrons of ordinary matter should melt into a plasma where the quark and gluon degrees of freedom are not anymore confined. This hot and dense primordial state of matter is called Quark Gluon Plasma (QGP) and should have existed in the expanding universe up to a few microseconds after the Big-Bang, when the phase transition to hadronic matter of confined quarks and gluons took place. Temperatures and energy densities similar to the ones in the very early universe can be created in collisions of heavy ions at ultra-relativistic energies in particle accelerators. Quantum Chromo Dynamics (QCD), an integral part of the Standard Mode, is the theory that describes the physics of the strong interaction. According to QCD, the phase transition should occur at a critical temperature of about $155 \mathrm{MeV}$, corresponding to a critical energy density $\epsilon$ of about $0.5 \mathrm{GeV} / \mathrm{fm}^{3}[1,2]$. In heavy-ion collisions at the energies of the Relativistic Heavy Ion Collider (RHIC) and at the Large Hadron Collider (LHC) values of $\epsilon$ that exceed by far this critical value are attained (e.g. $\epsilon \approx 14 \mathrm{GeV} / \mathrm{fm}^{3}$ as reported in [3] in $\mathrm{Pb}-\mathrm{Pb}$ collisions at the $\mathrm{LHC}$ ).

The aim of the heavy-ion program at both RHIC and LHC is to find convincing evidence of the existence of this new state of matter, to further study its properties and map the QCD phase diagram, usually depicted in terms of temperature $\mathrm{T}$ and baryochemical potential $\mu_{\mathrm{B}}$. The studies of anisotropic flow at high RHIC energies (i.e. in $\mathrm{Au}-\mathrm{Au}$ collisions at $\sqrt{s_{N N}}=130-200 \mathrm{GeV}$ ), that correspond to small $\mu_{\mathrm{B}}$ values [4-7] played fundamental role in revealing that the system behaves as an almost perfect liquid. This is reflected in the small value of the ratio of shear viscosity to

\footnotetext{
a e-mail: Panos.Christakoglou@nikhef.nl
}

entropy density $(\eta / s)$ which quantifies the friction of the created matter. In particular the value of $\eta / s$ is believed to be very close to the conjectured lower limit of $\hbar / 4 \pi k_{\mathrm{B}}$ [8]. The latest measurements at the LHC [9-14], where $\mu_{\mathrm{B}} \approx 0$, indicate that the system created in $\mathrm{Pb}-\mathrm{Pb}$ collisions at $\sqrt{s_{\mathrm{NN}}}=2.76 \mathrm{TeV}$ has similar characteristics.

In non-central nuclear collisions the initial spatial anisotropy of the overlap region between the two colliding nuclei is transformed into an anisotropy in momentum space through interactions between partons and at a later stage between the produced particles. Anisotropic flow characterises this final state momentum anisotropy and can be used to constrain the value of $\eta / s$. It can also provide valuable information about the initial state irregular shape of the overlap region of the colliding nuclei. These inhomogeneities result from the initial density profile of nucleons participating in the collision which is different from one event to the other [15-19].

Anisotropic flow studies are based on the description of the azimuthal distribution of particles by a Fourier series $d N / d \varphi \approx 1+\sum_{n=1}^{\infty} 2 v_{n} \cos \left[n\left(\varphi-\Psi_{n}\right)\right][20,21]$, where $\varphi$ is the azimuthal angle of particles, and $v_{n}=\left\langle\cos \left[n\left(\varphi-\Psi_{n}\right)\right]\right\rangle$ are the different flow coefficients developing relative to the symmetry planes $\Psi_{n}$ for each harmonic $n$. The second Fourier coefficient, $v_{2}$, measures the azimuthal momentum space anisotropy of particle emission relative to the second harmonic symmetry plane and is known as elliptic flow.

The study of elliptic flow of particle species provides the interesting possibility to probe physics mechanisms usually associated to different transverse momentum ranges in a more differential way. The first measurements of $v_{2}$ at RHIC energies [4-7] were described fairly well by ideal hydrodynamical calculations albeit up to low values of transverse momentum (i.e. for $p_{\mathrm{T}}<1.5 \mathrm{GeV} / c$ ). It was realised at a later stage that the inclusion of small viscous corrections extend the agreement with experimen- 
tal measurements to $p_{\mathrm{T}}<2.5 \mathrm{GeV} / c$. Although the development of elliptic flow at the partonic stage of the evolution of the system is a well established concept, it is crucial to further understand the role and the relevant significance of the hadronic rescattering phase. This can be done by studying particles, such as $\phi, \Xi^{-}+\bar{\Xi}^{+}$and $\Omega^{-}+\bar{\Omega}^{+}$, with a small hadronic cross-section which are expected to probe mainly the early, partonic stages of the collision.

In the intermediate transverse momentum region $(2<$ $\left.p_{\mathrm{T}}<8 \mathrm{GeV} / c\right)$ an interesting scaling emerged at RHIC [22-24]: when $v_{2}\left(p_{\mathrm{T}}\right)$ scaled by the number of constituent quarks, $n_{q}$, (i.e. 2 for mesons and 3 for baryons) is plotted as a function of $p_{\mathrm{T}} / n_{q}$, all particle species seem to be lying in one universal curve for $p_{\mathrm{T}} / n_{q}>1 \mathrm{GeV} / c$. This is usually referred to as number of constituent quarks (NCQ) scaling, and led naturally to the development of a picture where flow develops at the partonic level and particles are created via coalescence of constituent quarks [25-29].

At high values of transverse momentum (i.e. for $p_{\mathrm{T}}>$ $8 \mathrm{GeV} / c$ ), particles are predominantly produced via the fragmentation of energetic partons. These partons, interact with the medium while traversing it and experience radiative and collisional energy loss [30, 31]. This mechanism develops an azimuthal asymmetry in the presence of an initially asymmetric system, like the one in non-central collisions: partons crossing the short axis (i.e. in plane) of the overlap region, cross smaller path in the medium and thus their energy is less attenuated compared to the ones that cross the long axis (i.e. out of plane). This leads to an anisotropy in the particle production and the development of $v_{2}$ that can now be used to probe the path length dependence of energy loss [32,33].

In the discussion of the $v_{2}$ in these different transverse momentum ranges, the presence of the partonic stage in the evolution of the system is of fundamental importance. One interesting possibility to experimentally control the formation of the QGP is provided by moving to large values $\mu_{\mathrm{B}}$. This can be done by lowering the collision energy in fine steps, and thus mapping the QCD phase diagram. This is done in the Beam Energy Scan (BES) program of RHIC, where Au-Au collisions at $\sqrt{s_{N N}}=62.4,39,27$, $19.5,11.5$ and $7.7 \mathrm{GeV}$ are recorded. The study of elliptic flow at these lower energies can give valuable indications as to whether the hadronic instead of partonic degrees of freedom become relevant.

In this article, I review the latest experimental results on the elliptic flow for identified particles, from heavy-ion collisions at both RHIC [34, 35] and LHC [36].

\section{Azimuthal anisotropy at low $\mu_{\mathrm{B}}$}

In [36] the ALICE Collaboration presented the $p_{\mathrm{T}}$ differential $v_{2}$ (i.e. $v_{2}\left(p_{\mathrm{T}}\right)$ ) of mesons $\left(\pi^{ \pm}, \mathrm{K}^{ \pm}, \mathrm{K}_{\mathrm{S}}^{0}, \phi\right)$ and baryons (p, $\Lambda, \Xi^{-}, \Omega^{-}$, and their antiparticles) measured in $\mathrm{Pb}-\mathrm{Pb}$ collisions at $\sqrt{s_{\mathrm{NN}}}=2.76 \mathrm{TeV}$. The results were reported in $|y|<0.5$, with $y$ the rapidity of each particle, and $0.2<p_{\mathrm{T}}<6.0 \mathrm{GeV} / c$. The values of $v_{2}$ were obtained with the Scalar Product method [45, 46], using a pseudo-rapidity gap of $|\Delta \eta|>0.9$ to suppress correlations

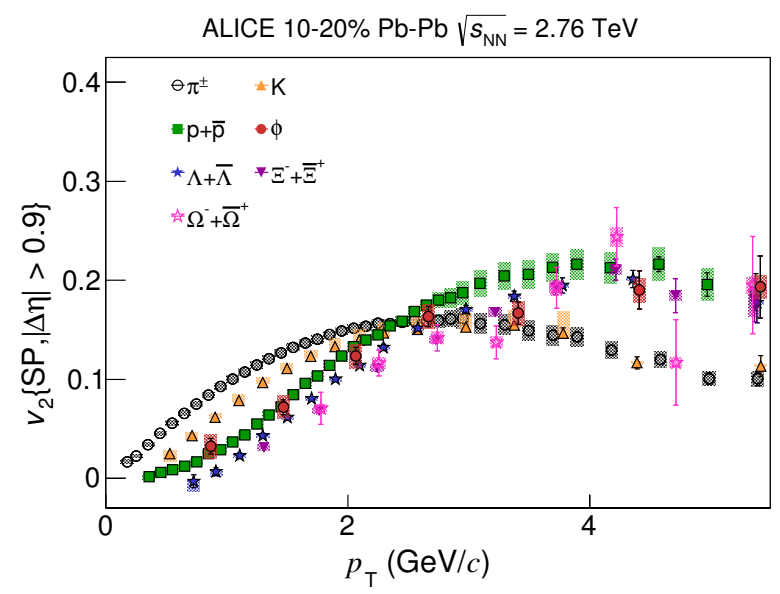

Figure 1. The $p_{\mathrm{T}}$-differential $v_{2}$ for different particle species in the $10-20 \%$ centrality interval of $\mathrm{Pb}-\mathrm{Pb}$ collisions at $\sqrt{s_{\mathrm{NN}}}=$ $2.76 \mathrm{TeV}$. The data points are extracted from [36].

not related to the common symmetry plane. The latter are known as non-flow effects and consist of correlations arising from jets or resonance decays and quantum statistics correlations.

Figure 1 presents the dependence of $v_{2}$ for different particle species on transverse momentum in the 10$20 \%$ centrality interval of $\mathrm{Pb}-\mathrm{Pb}$ collisions at $\sqrt{s_{\mathrm{NN}}}=$ $2.76 \mathrm{TeV}$. For $p_{\mathrm{T}}<2 \mathrm{GeV} / c$ particles are ordered according to their mass i.e. for a fixed value of $p_{\mathrm{T}}$ heavier particles exhibit lower $v_{2}$ compared to lighter particles. This ordering is induced by an interplay between radial and elliptic flow that results in a mass dependent lower $v_{2}$ value [36]. This can be understood considering that radial flow acts over the particle spectra that consist of two components i.e. the thermal part and the part that is affected by the expanding medium and is mass dependent. This mass dependent component introduces a depletion in the particle spectrum at low values of $p_{\mathrm{T}}$, which is more pronounced for heavier particles. In an azimuthally asymmetric system, the pressure gradient and thus the expansion velocity is larger in-plane compared to the out-of-plane direction. This naturally leads to a larger depletion at low values of $p_{\mathrm{T}}$ in the direction of the symmetry plane. Since $v_{2}$ quantifies the relative difference of particle yield inplane versus out-of-plane, this mechanism leads naturally to a reduction of the value of $v_{2}$. When the two effects (i.e. radial flow acting on particle spectra and an azimuthally asymmetric system) are coupled then $v_{2}$ becomes lower with increasing mass for a given value of $p_{\mathrm{T}}$.

Beyond the crossing point and at the intermediate $p_{\mathrm{T}}$ (i.e. for $2<p_{\mathrm{T}}<6 \mathrm{GeV} / c$ ), particles seem to form two different groups i.e. one for baryons and one for mesons. The particle type grouping and the implications to the coalescence formalism will be discussed in Section 2.2. Looking into the details of fig. 1 already at this stage, one can notice that the $\phi$-meson already breaks this grouping by following the baryon band, at least in this centrality interval. 


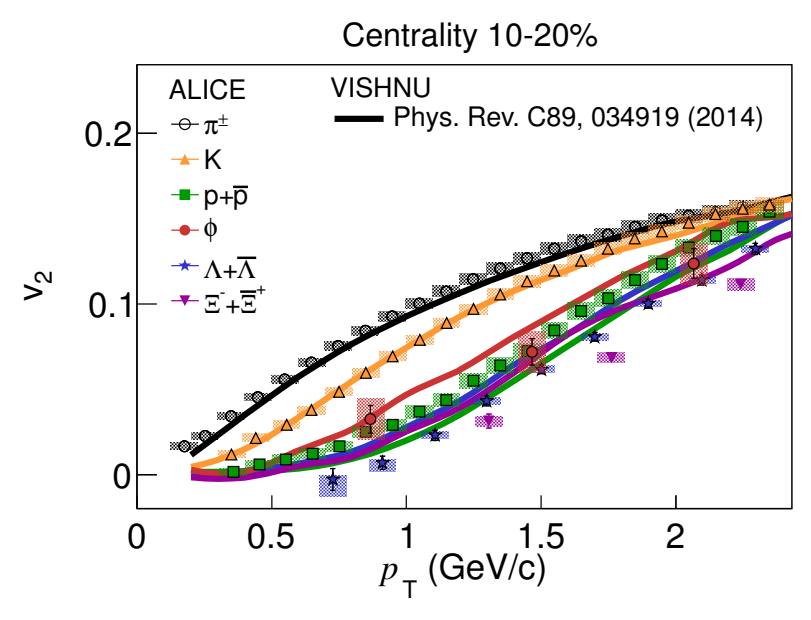

Figure 2. The $p_{\mathrm{T}}$-differential $v_{2}$ for different particle species in the $10-20 \%$ centrality interval of $\mathrm{Pb}-\mathrm{Pb}$ collisions at $\sqrt{s_{\mathrm{NN}}}=$ $2.76 \mathrm{TeV}$. The experimental data points from [36] are compared with VISHNU calculations [37-39], which couples the hydrodynamical evolution of the system with a hadron cascade model.

\subsection{Test of hydrodynamical picture}

After the initial success of ideal hydrodynamical calculations in describing the integrated and differential $v_{2}$, new theoretical calculations emerged incorporating the effects of viscous corrections. However recently, there were attempts to couple the hydrodynamical evolution of the system to a hadronic cascade model. Such calculations are important to be confronted to data to unveil the importance of the hadronic rescattering phase in the development of $v_{2}$. Such comparisons can also play a significant role in tuning the input parameters (e.g. hadronic cross-sections) for the different stages.

Figure 2 presents the comparison of the experimental measurements for central events with the calculations extracted from [37-39]. These calculations are based on VISHNU that couples the hydrodynamical evolution of the system to a hadronic cascade model. VISHNU provides a reasonable description of the transverse momentum dependence of $v_{2}$ for $\pi^{ \pm}$and K. However, for heavier particles one can observe significant differences. In particular, the model significantly underestimates the elliptic flow for protons, while overestimating $v_{2}$ of $\phi, \Lambda+\bar{\Lambda}$ and $\Xi^{-}+\bar{\Xi}^{+}$. What is striking though, is that VISHNU expects that the mass ordering is not preserved, something which is not supported by the experimental measurements [36].

One natural question is whether this breaking of mass ordering is induced by the hadronic cascade, which follows the hydrodynamical evolution of the system. In an attempt to answer this question, fig. 3 presents the comparison between the $p_{\mathrm{T}}$-differential $v_{2}$ from VISHNU and VISH2+1 [43]. The latter is a 2+1-dimension pure hydrodynamical calculation, with a non-zero shear viscosity over entropy density ratio. The same centrality interval as in fig. 2 is reported for three indicative particle species for each plot: $\pi^{ \pm}, \mathrm{p}+\overline{\mathrm{p}}$ and $\phi$ in the upper and $\mathrm{K}^{ \pm}, \Lambda+\bar{\Lambda}$
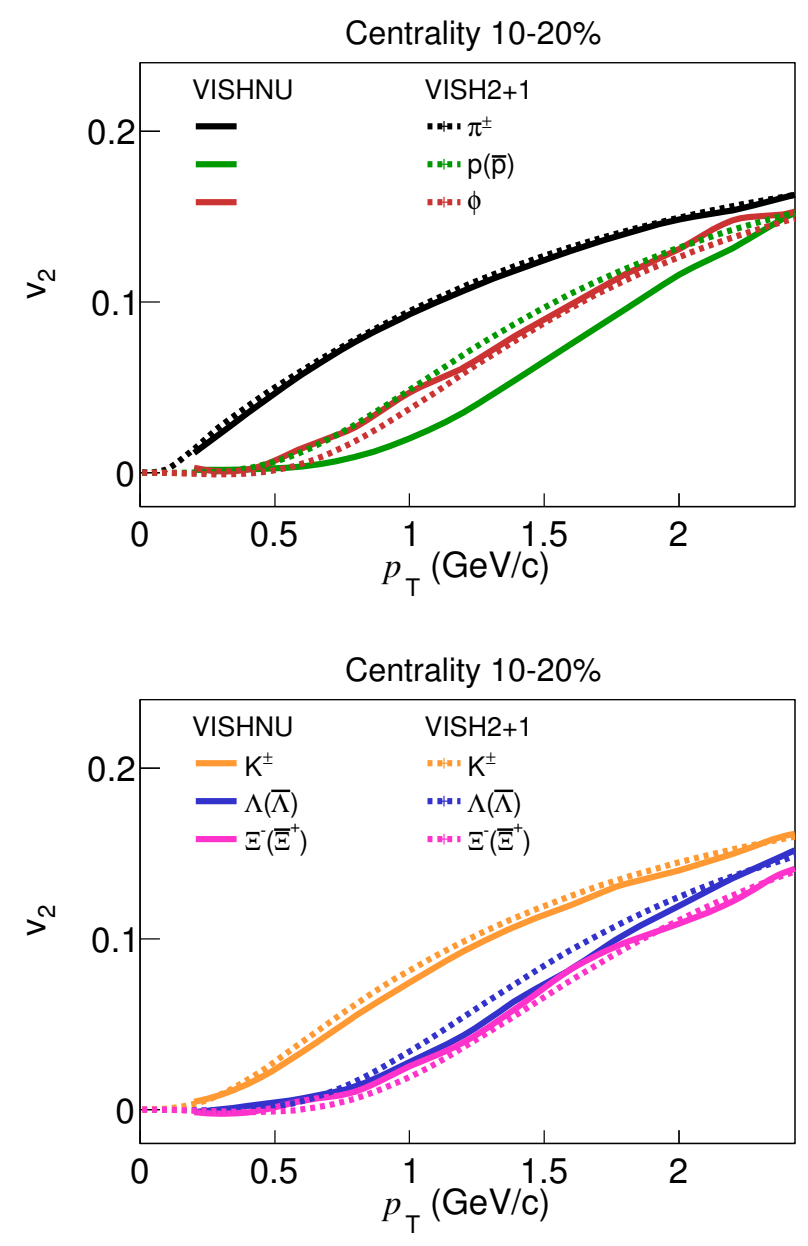

Figure 3. Comparison between calculations from VISHNU (solid lines) [37-39] and VISH2+1 (dashed lines) [43] for the 10$20 \%$ centrality interval of $\mathrm{Pb}-\mathrm{Pb}$ collisions at $\sqrt{s_{\mathrm{NN}}}=2.76 \mathrm{TeV}$. The results for $\pi^{ \pm}, \mathrm{p}+\overline{\mathrm{p}}, \phi$, and $\mathrm{K}^{ \pm}, \Lambda+\bar{\Lambda}$ and $\Xi^{-}+\bar{\Xi}^{+}$are presented in the upper and lower plot, respectively.

and $\Xi^{-}+\bar{\Xi}^{+}$in the lower plot. It is seen that VISHNU, represented by the solid curves, clearly violates the mass ordering, as exhibited e.g. by the relevant lines for $\mathrm{p}+\overline{\mathrm{p}}$ and $\phi$ (green and red, respectively). On the other hand, the dashed lines that correspond to VISH2+1 exhibit similar mass ordering as the one observed in the measurements.

Furthermore, it is of great interest to note that while the curves for the two different calculations for $\pi^{ \pm}$are quite similar, there is a significant difference observed for $\mathrm{p}+\overline{\mathrm{p}}$. In particular, VISHNU expects stronger radial flow than VISH2 +1 , which is reflected in lower $v_{2}$ for fixed transverse momentum values. Moreover, it is also worth noticing that the inclusion of the hadronic phase for the case of the $\phi$-meson does not introduce any significant deviation in $v_{2}\left(p_{\mathrm{T}}\right)$ as compared to the pure hydrodynamical calculation. This could be related to the small $\phi$ hadronic crosssection that is used as an input in VISHNU. Finally, for other particles containing s-quarks, it is seen that the inclusion of the hadronic rescattering phase introduces only marginal differences for $\mathrm{K}^{ \pm}$and $\Lambda+\bar{\Lambda}$. For both cases the 

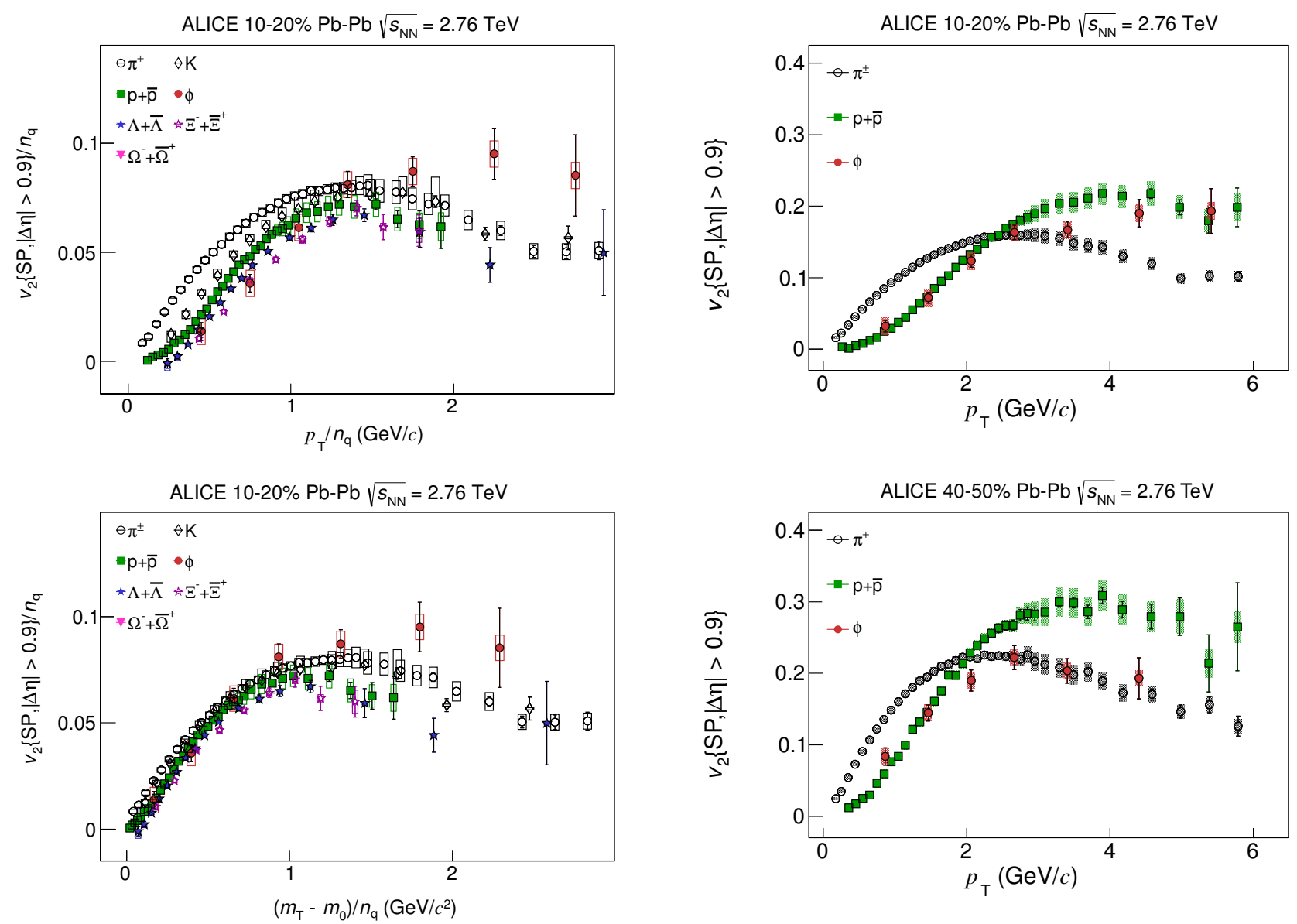

Figure 4. The $p_{\mathrm{T}} / n_{q}$ (upper plot) and $\left(m_{\mathrm{T}}-m_{0}\right) / n_{q}$ (lower plot) dependence of $v_{2} / n_{q}$ for $\pi^{ \pm}, \mathrm{K}, \mathrm{p}+\overline{\mathrm{p}}, \phi, \Lambda+\bar{\Lambda}$, and $\Xi^{-}+\bar{\Xi}^{+}$for $\mathrm{Pb}-\mathrm{Pb}$ collisions in the $10-20 \%$ centrality intervals of $\mathrm{Pb}-\mathrm{Pb}$ collisions at $\sqrt{s_{\mathrm{NN}}}=2.76 \mathrm{TeV}$. The data points are extracted from [36].

$v_{2}$ values obtained in VISHNU are lower than the ones from VISH $2+1$ for a fixed $p_{\mathrm{T}}$. On the other hand, for $\Xi^{-}+\bar{\Xi}^{+}$there is no significant difference between these two calculations and if there is any it works in the opposite direction i.e. the $v_{2}$ at a given $p_{\mathrm{T}}$ in VISH2 +1 is smaller than VISHNU. This decrease can be attributed to the small hadronic cross-section that the $\Xi^{-}+\bar{\Xi}^{+}$are estimated to have.

In conclusion, it seems that the development of flow is affected by the inclusion of the hadronic resctattering phase. This influence seems to have a dependence on the quark flavor, with particles containing s-quarks being significantly less affected as compared to hadrons consisting of light quarks.

\subsection{Test of scaling properties}

At the intermediate $p_{\mathrm{T}}$ range (i.e. $2<p_{\mathrm{T}}<8 \mathrm{GeV} / c$ ), coalescence is believed to be the dominant particle production mechanism. This picture was reinforced by the observation that at RHIC energies particles tend to follow a simple scaling law: different particle species follow, within

Figure 5. The $p_{\mathrm{T}}$-differential $v_{2}$ for $\pi^{ \pm}, \mathrm{p}+\overline{\mathrm{p}}$ and $\phi$-mesons, in the $10-20 \%$ (upper plot) and he 40-50\% (lower plot) centrality intervals of $\mathrm{Pb}-\mathrm{Pb}$ collisions at $\sqrt{s_{\mathrm{NN}}}=2.76 \mathrm{TeV}$. The data points are extracted from [36].

uncertainties, a universal curve when $v_{2} / n_{q}$ is plotted as a function of $p_{\mathrm{T}} / n_{q}$, for $p_{\mathrm{T}} / n_{q}>1 \mathrm{GeV} / c$ [22-24]. To extend this scaling to lower transverse momentum values, the PHENIX collaboration in $[23,24]$ proposed to use the transverse kinetic energy defined as $K E_{\mathrm{T}}=m_{\mathrm{T}}-m_{0}$ as a scaling variable since it accounts for the blue shift induced by radial flow.

The upper plot of fig. 4 presents the $p_{\mathrm{T}} / n_{q}$ dependence of $v_{2} / n_{q}$ for different particle species measured in central $\mathrm{Pb}-\mathrm{Pb}$ collisions at $\sqrt{s_{N N}}=2.76 \mathrm{TeV}$. It is seen that there are deviations from the universal scaling of around $\pm 20 \%$ for $p_{\mathrm{T}} / n_{q}>1 \mathrm{GeV} / c$. Similar deviations are observed in the lower plot of fig. 4 , where $v_{2} / n_{q}$ is plotted as a function of $\left(m_{\mathrm{T}}-m_{0}\right) / n_{q}$ for the same centrality interval and for $\left(m_{\mathrm{T}}-m_{0}\right) / n_{q}>0.6-0.8 \mathrm{GeV} / c^{2}$. No significant difference compared to this picture is reported in [36] for other centrality intervals.

\subsection{The important role of the $\phi$-meson}

The case of the $\phi$-meson is of great interest in all these studies. Its mass lies between the one of protons and $\Lambda$ and thus provides the possibility to investigate experimen- 
tally whether the mass ordering is preserved at low values of $p_{\mathrm{T}}$. In addition, due to its small hadronic crosssection [48], it is expected to probe mainly the partonic stages of the collision and in parallel give valuable feedback to hybrid hydrodynamical approaches that attempt to couple the hadronic cascade phase to the early, hydrodynamical evolution of the system. Finally, it provides a testing ground of the baryon-meson grouping at intermediate values of $p_{\mathrm{T}}$.

Figure 5 presents the transverse momentum dependence of $v_{2}$ for charged pions (open black circles), $\mathrm{p}+\overline{\mathrm{p}}$ (green filled squares) and $\phi$ (red filled circles) measured in $\mathrm{Pb}-\mathrm{Pb}$ collisions at $\sqrt{s_{N N}}=2.76 \mathrm{TeV}$. Two centrality intervals are shown: the $10-20 \%$ and the $40-50 \%$ intervals on the left and right plot, respectively. It is seen that at low values of transverse momentum the $v_{2}$ of the $\phi$-meson is slightly smaller than the value of $\mathrm{p}+\overline{\mathrm{p}}$ at a given value of $p_{\mathrm{T}}$. However, the lowest transverse momentum point of $\phi$ for both centralities might be hinting to a reverse mass ordering. The current statistical and systematic uncertainty extracted from the analyzed data sample does not allow to reach a solid conclusion. This can be further explored with either the analysis of the 2011 sample or with the new data set that ALICE is going to collect in run-II.

At the intermediate transverse momentum region, the $\phi$-meson seems to be following the baryon band for central collisions, while progressively moving to the meson band in peripheral events. This indicates that at least in central events the mass and not the number of constituent quarks is still the driving force of the behavior of elliptic flow. This surprising behavior of the $\phi$-meson can not be accommodated within the coalescence picture and seems to request for a refinement of its formalism.

\subsection{Comparison between LHC and RHIC energies}

One of the interesting observations of the first measurements at the LHC [9] was that although the $p_{\mathrm{T}}$-differential $v_{2}$ for several centrality intervals was not only qualitatively but also quantitatively in agreement with top RHIC energies, the integrated values are larger by $30 \%$. This was attributed to a larger $\left\langle p_{\mathrm{T}}\right\rangle$ at the LHC as compared to RHIC. To study this reported similarity in $v_{2}\left(p_{\mathrm{T}}\right)$, and answer the natural question of whether this was accidental or it has some further physics implications, it is instructive to look at the relevant comparison of $v_{2}$ for identified particles.

Figure 6 presents the $p_{\mathrm{T}}$-differential $v_{2}$ for the $10 \%$ most central heavy-ion collisions at LHC and at RHIC, the latter for Au-Au collisions at $\sqrt{s_{N N}}=62.4 \mathrm{GeV}[34,35]$. Since there are differences in the $v_{2}$ values between particles and antiparticles at RHIC energies, that will be discussed in Section 3, the results for both are shown separately. In the low transverse momentum region (i.e. for $p_{\mathrm{T}}<2 \mathrm{GeV} / c$ ), it is seen that the experimental points for charged pions at the LHC are systematically above the relevant values at RHIC, albeit not significantly. On the other hand, the data points for protons indicate clear and significant differences. In particular, the green squares that represent the results at the $\mathrm{LHC}$ exhibit lower $v_{2}$ at a fixed

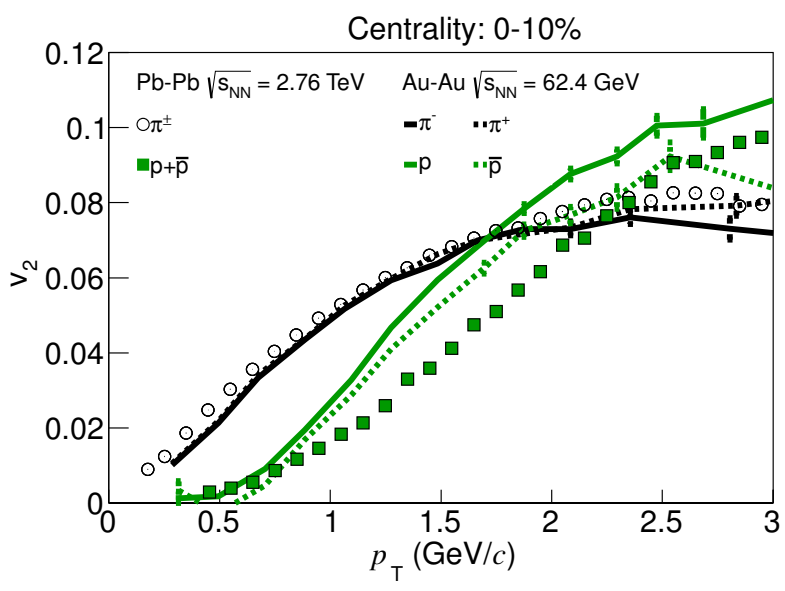

Figure 6. The comparison of the $p_{\mathrm{T}}$-differential $v_{2}$ for $\pi^{ \pm}$and $\mathrm{p}+\overline{\mathrm{p}}$ for the $10 \%$ most central $\mathrm{Pb}-\mathrm{Pb}$ and $\mathrm{Au}-\mathrm{Au}$ collisions at the LHC and RHIC, respectively. The LHC and RHIC points are extracted from [36] and [34, 35], respectively.

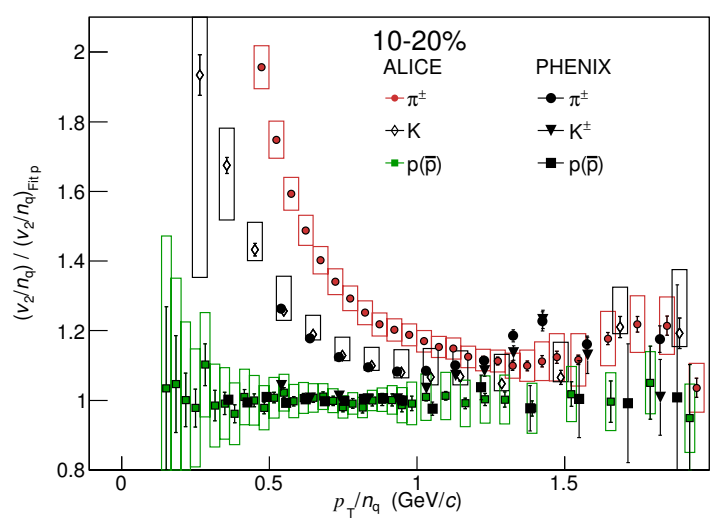

Figure 7. The $p_{\mathrm{T}} / n_{q}$ dependence of the double ratio of $v_{2} / n_{q}$ for $\pi^{ \pm}, \mathrm{K}$ and $\mathrm{p}+\overline{\mathrm{p}}$ relative to a fit to $v_{2} / n_{q}$ of $\mathrm{p}$ and $\overline{\mathrm{p}}$ in $\mathrm{Pb}-\mathrm{Pb}$ and $\mathrm{Au}-\mathrm{Au}$ collisions at $\sqrt{s_{\mathrm{NN}}}=2.76 \mathrm{TeV}$ [36] and $200 \mathrm{GeV}$ [47], respectively.

value of $p_{\mathrm{T}}$ than the solid (protons) and dashed (antiprotons) green lines measured at RHIC. This is in agreement with the larger value of radial flow that is measured at $\sqrt{s_{N N}}=2.76 \mathrm{TeV}$ as compared to the energy ranges that RHIC covers [44].

At the intermediate transverse momentum range, to quantify and compare the deviations in the NCQ scaling between LHC and RHIC, one can calculate the double ratio of $v_{2} / n_{q}$ for $\pi^{ \pm}, \mathrm{K}$ and $\mathrm{p}+\overline{\mathrm{p}}$ relative to a fit to $v_{2} / n_{q}$ of $\mathrm{p}$ and $\overline{\mathrm{p}}$ for both the LHC and RHIC energies. This double ratio is then plotted as a function of $p_{\mathrm{T}} / n_{q}$ for the $10-20 \%$ centrality interval for both $\mathrm{Pb}-\mathrm{Pb}$ and $\mathrm{Au}-\mathrm{Au}$ collisions at $\sqrt{s_{N N}}=2.76 \mathrm{TeV}$ and $200 \mathrm{GeV}$, respectively, in fig. 7. The RHIC data points are extracted from [47]. It is seen that the deviations at intermediate values of transverse momen- 


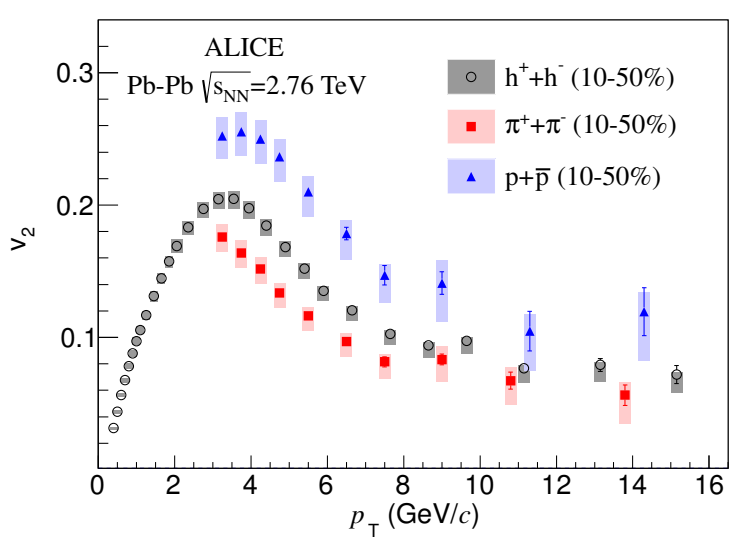

Figure 8. The transverse momentum dependence of $v_{2}$ of unidentified charged particles, charged pions and protons for the 10$50 \%$ centrality interval, reported in $\mathrm{Pb}-\mathrm{Pb}$ collisions at $\sqrt{s_{N N}}=$ $2.76 \mathrm{TeV}$. The data points are extracted from [49]

tum are qualitatively but also quantitatively similar at the two energy regimes. Finally, it is interesting to note that there are differences in the $p_{\mathrm{T}} / n_{q}$ evolution of this double ratio for $\pi^{ \pm}$and K between ALICE [36] and PHENIX [47].

\subsection{Fragmentation region}

At high values of transverse momentum (i.e. for $p_{\mathrm{T}}>$ $8 \mathrm{GeV} / \mathrm{c}$ ), azimuthal anisotropy is introduced by the geometry of the system in conjunction with energetic partons traversing along either the short (i.e. in-plane) or the long axis (i.e. out-of-plane) of the fireball. Partons moving in-plane traverse a smaller path in the medium than when moving out-of-plane and thus have smaller probability to be absorbed or have their energy attenuated. The measurement of $v_{2}$ can thus be used to probe the path length dependence of energy loss.

Figure 8 presents the transverse momentum dependence of $v_{2}$ in the $10-50 \%$ centrality interval in $\mathrm{Pb}-\mathrm{Pb}$ collisions at $\sqrt{s_{N N}}=2.76 \mathrm{TeV}$ for charged particles (black markers), identified pions (red markers) and protons (blue markers). For $3<p_{\mathrm{T}}<8 \mathrm{GeV} / c$ the values of $v_{2}$ decrease dramatically. In this transverse momentum range the markers representing the results for charged hadrons lie between the points for protons and pions. For $p_{\mathrm{T}}>8 \mathrm{GeV} / c$ the decrease seems not to be significant. In addition, for $p_{\mathrm{T}}>10 \mathrm{GeV} / c$ it is seen that there is no significant difference in $v_{2}$ for pions and protons within the reported uncertainties. This is in agreement with observations of no significant particle species dependence of the nuclear modification factor, as reported in [49].

\section{Azimuthal anisotropy at high $\mu_{\mathrm{B}}$}

By lowering the center-of-mass energy it is expected that the system will spend less time in the partonic stage. In addition, if the energy is low enough, the system might not even form a deconfined phase. Indications about this transition to a phase where the hadronic over the partonic stage becomes progressively dominant can be provided by studying whether the main features of $v_{2}$ measurements (e.g. mass ordering, NCQ scaling) observed at low values of $\mu_{\mathrm{B}}$ are also present at lower energies. The Beam Energy Scan (BES) is performed by recording $\mathrm{Au}-\mathrm{Au}$ collisions at $\sqrt{s_{N N}}=62.4,39,27,19.5,11.5$ and $7.7 \mathrm{GeV}$, thus mapping the QCD phase diagram at relatively high values of $\mu_{\mathrm{B}}$. Currently, BES is one of the priorities of the heavyion physics program at RHIC.

Figure 9 presents the transverse momentum dependence of $v_{2}$ for particles at different energies for the $80 \%$ most central $\mathrm{Au}-\mathrm{Au}$ collisions [34, 35]. It is seen that particles are ordered according to their mass for all energies in agreement with hydrodynamical calculations. It is interesting to note that the spread of the data points for a fixed value of $p_{\mathrm{T}}$ decreases with decreasing energy. This observation could be related to a decreased contribution from radial flow with decreasing energy. Among all particles, the $\phi$-meson seems to behave differently, i.e. by not following the mass ordering trend, starting from $\sqrt{s_{N N}}=39 \mathrm{GeV}$. Considering the small hadronic crosssection that this particle is believed to have, this observation could be attributed to a progressively more dominant role of the hadronic over the partonic stage with decreasing beam energy.

Figure 10 presents the similar picture as in fig. 9 but this time for antiparticles. Also in this case, the mass ordering at this low transverse momentum range is visible. However, contrary to the picture that emerged for particles, the spread of the data points of $v_{2}$ for a given $p_{\mathrm{T}}$ does not decrease with decreasing energy.

The comparison of $v_{2}$ values for both particles and antiparticles reveal an interesting, significant quantitative difference. This is illustrated in fig. 11 that presents the $p_{\mathrm{T}}$ independent difference of $v_{2}$ between particles and antiparticles as a function of center-of-mass energy. With the exception of pions where the effect is reversed, all other particles exhibit larger $v_{2}$ values as compared to the corresponding antiparticles. These differences become larger with decreasing energy. Contributions to this evident energy dependence of $v_{2}(X)-v_{2}(\overline{\mathrm{X}})$ can originate from the increasing baryon stopping at mid-rapidity with decreasing energy. However, currently it is not clear whether these effects can quantitatively describe the observed differences. Further theoretical calculations can shed light to the origin of this interesting effect that emerges at high values of $\mu_{\mathrm{B}}$.

\section{Summary and outlook}

The studies of anisotropic flow continue to play an important role in improving our understanding of the behavior of matter created in a collision of heavy ions. In these proceedings results from measurements of the second Fourier coefficient $v_{2}$ for identified particles from two regions of the QCD phase diagram were discussed.

At low values of baryochemical potential $\mu_{\mathrm{B}}$, the created system behaves as an almost perfect liquid and the relevant degrees of freedom are of partonic nature. For $p_{\mathrm{T}}<2 \mathrm{GeV} / c$, a clear mass ordering is reported at both 


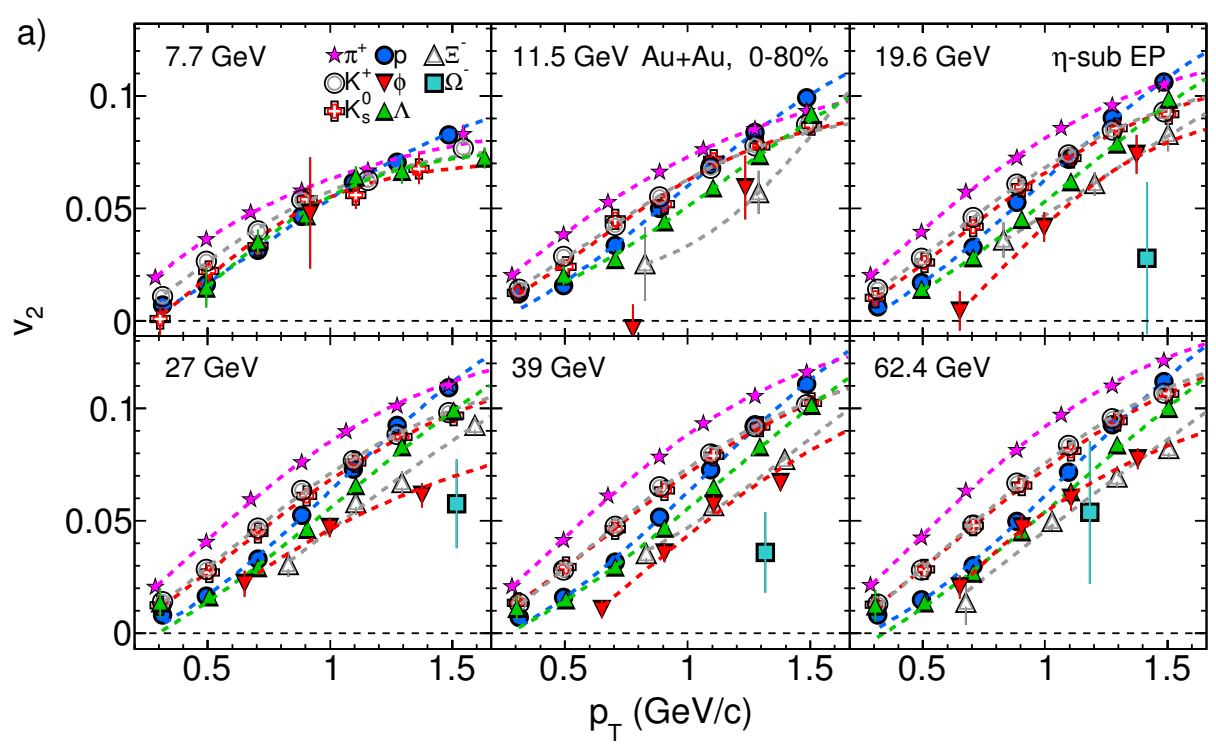

Figure 9. The transverse momentum dependence of $v_{2}$ in the $0-80 \%$ central $\mathrm{Au}+\mathrm{Au}$ collisions at $\sqrt{s_{N N}}=62.4 \mathrm{GeV}$ for different particles. Each plot corresponds to a different energy, recorded during the BES at RHIC. The data points are extracted from [34, 35].

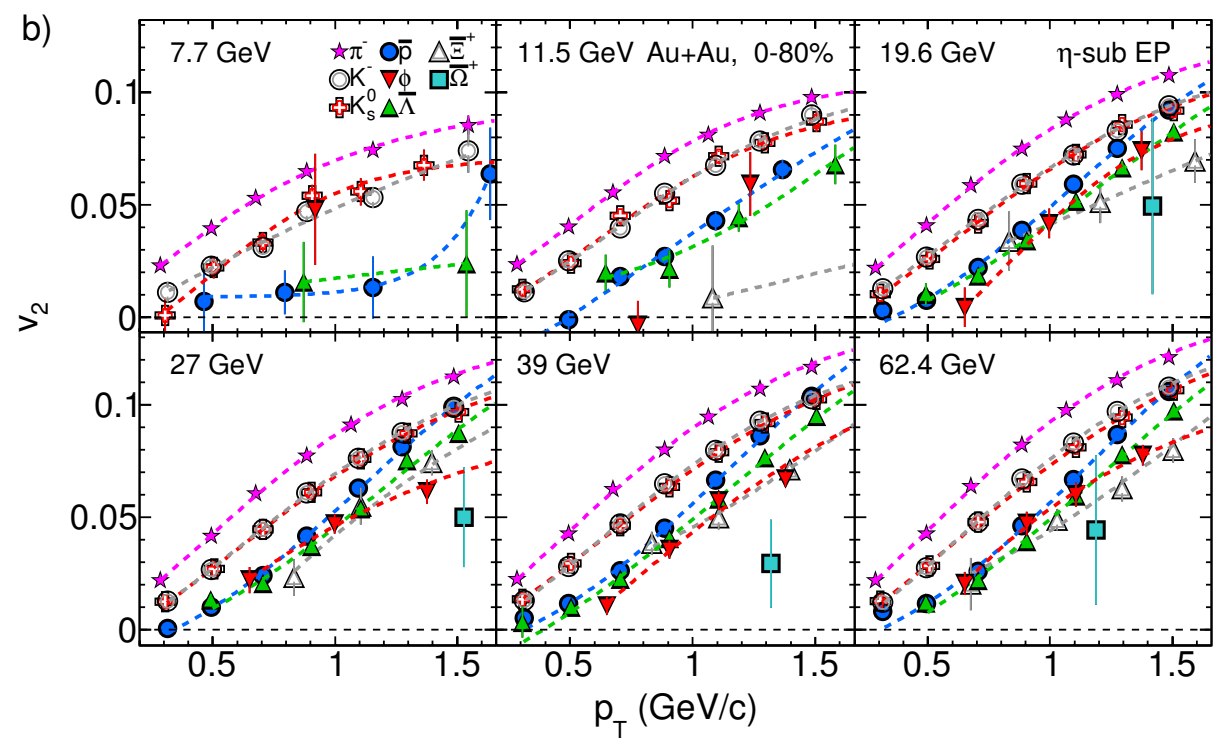

Figure 10. The transverse momentum dependence of $v_{2}$ in the $0-80 \%$ central $\mathrm{Au}+\mathrm{Au}$ collisions at $\sqrt{s_{N N}}=62.4 \mathrm{GeV}$ for different antiparticles. Each plot corresponds to a different energy, recorded during the BES at RHIC. The data points are extracted from [34, 35].

RHIC and LHC, that originates from the interplay between radial and elliptic flow. The comparison with hydrodynamical calculations coupled to a hadron cascade model (i.e. VISHNU) revealed interesting features. According to VISHNU, radial and elliptic flow develops during the hadronic stage in a quark flavor dependent way, inducing a breaking of mass ordering. This breaking is not supported by the ALICE measurements. For $2<p_{\mathrm{T}}<6 \mathrm{GeV} / c$ the number of constituent quark scaling at the LHC seems to hold at no better than $\pm 20 \%$. Similar deviations though were observed already in Au-Au collisions at $\sqrt{s_{N N}}=$ $200 \mathrm{GeV}$. The $\phi$-meson, an important probe due to its mass and quark content, provides a surprising picture at this momentum range: the corresponding data points follow the band of baryons for central events while they move progressively to the meson band for peripheral events. This seems to indicate that the mass and not the number of constituent quarks is the key ingredient for the development of 


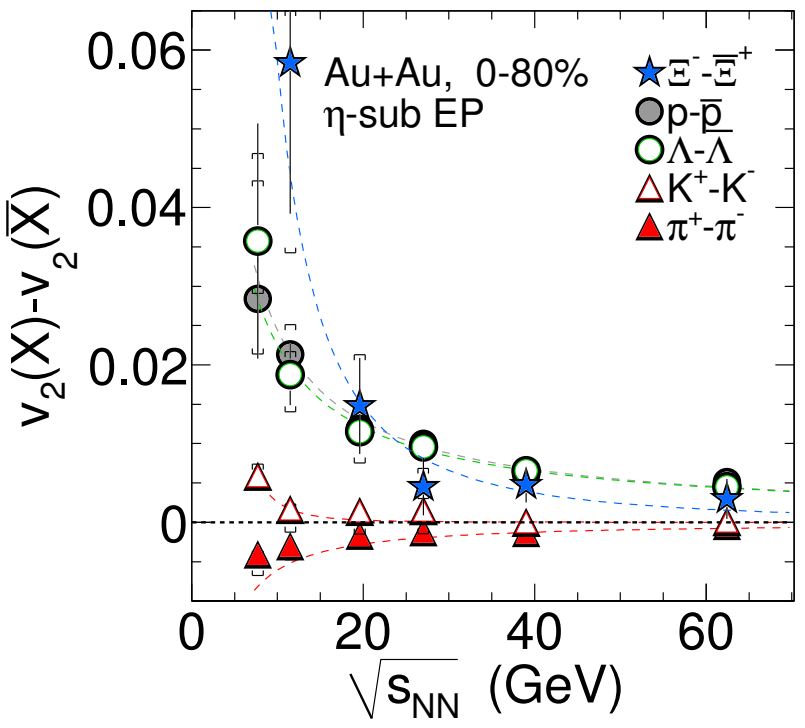

Figure 11. The difference in $v_{2}$ between particles and their corresponding anti-particles, depicted as $v_{2}(X)-v_{2}(\overline{\mathrm{X}})$ ) as a function of $\sqrt{s_{N N}}$ for $0-80 \%$ central $\mathrm{Au}+\mathrm{Au}$ collisions. The data points are extracted from $[34,35]$.

$v_{2}$, at least for central events. For $p_{\mathrm{T}}<10 \mathrm{GeV} / c$, where one can probe the path length dependence of energy loss, non-zero $v_{2}$ values are reported that exhibit no significant particle species dependence.

At the beam energy scan program of RHIC, by increasing the values of $\mu_{\mathrm{B}}$, one can reach conditions where the hadronic over the partonic degrees of freedom become dominant. Although for both particles and antiparticles a mass ordering is observed for all energies, the spread of $v_{2}$ at a fixed value of transverse momentum decreases with decreasing energy for particles, while for antiparticles the changes (if any) are not significant. Also at these energy ranges, the $\phi$-meson plays a key role in the development of the picture that emerges, since it violates the mass ordering. This observation, coupled to the small hadronic cross-section that this particle is believed to have, could be indicative of the transition to a system where the hadronic stage becomes progressively dominant.

The study of different harmonics of anisotropic flow is expected to play a significant role during the second run of the LHC data-taking, starting in 2015, but also during the continuation of the beam energy scan at RHIC. They will allow not only to constrain further the value of $\eta / s$ and the initial conditions of heavy-ion collisions, but will also assist in establishing the role of the hadronic rescattering phase in the development of flow. Finally, with the extension of these studies in smaller systems using multiparticle techniques, one could try to answer whether we have already observed the onset of collective effects in high multiplicity pp [50], p- $\mathrm{Pb}$ [51-57] and d-Au [58] collisions and try to probe their hydrodynamical nature.

\section{Acknowledgements}

I am grateful to the people listed below for the stimulating discussions, the contributions to the figures and for improvement on the text: Frank Geerts, Paul Kuijer, Francesco Noferini, Carlos Perez, Jurgen Schukraft, Raimond Snellings, Sergei Voloshin, Hui Wang, Zhongbao Yin and You Zhou.

\section{References}

[1] S. Borsanyi, G. Endrodi, Z. Fodor, A. Jakovac, S. D. Katz, S. Krieg, C. Ratti and K. K. Szabo, JHEP 1011 (2010) 077 [arXiv:1007.2580 [hep-lat]].

[2] T. Bhattacharya, M. I. Buchoff, N. H. Christ, H. T. Ding, R. Gupta, C. Jung, F. Karsch and Z. Lin et al., arXiv:1402.5175 [hep-lat].

[3] S. Chatrchyan et al. [CMS Collaboration], Phys. Rev. Lett. 109 (2012) 152303 [arXiv:1205.2488 [nucl-ex]].

[4] I. Arsene et al. [BRAHMS Collaboration], Nucl. Phys. A 757 (2005) 1 [nucl-ex/0410020].

[5] K. Adcox et al. [PHENIX Collaboration], Nucl. Phys. A757 (2005) 184, nucl-ex/0410003.

[6] B. B. Back et al. [PHOBOS Collaboration], Nucl. Phys. A757 (2005) 28, nucl-ex/0410022.

[7] J. Adams et al. [STAR Collaboration], Nucl. Phys. A757 (2005) 102, nucl-ex/0501009.

[8] P. Kovtun, D. T. Son and A. O. Starinets, Phys. Rev. Lett. 94 (2005) 111601 [hep-th/0405231].

[9] K. Aamodt et al. [ALICE Collaboration], Phys. Rev. Lett. 105 (2010) 252302 [arXiv:1011.3914 [nucl-ex]].

[10] G. Aad et al. [ATLAS Collaboration], Phys. Rev. C 86 (2012) 014907 [arXiv:1203.3087 [hep-ex]].

[11] S. Chatrchyan et al. [CMS Collaboration], Phys. Rev. C 87 (2013) 014902 [arXiv:1204.1409 [nucl-ex]].

[12] K. Aamodt et al. [ALICE Collaboration], Phys. Rev. Lett. 107 (2011) 032301 [arXiv:1105.3865 [nucl-ex]].

[13] G. Aad et al. [ATLAS Collaboration], JHEP 1311 (2013) 183 [arXiv:1305.2942 [hep-ex]].

[14] S. Chatrchyan et al. [CMS Collaboration], Phys. Rev. C 89 (2014) 044906 [arXiv:1310.8651 [nucl-ex]].

[15] R. S. Bhalerao and J. -Y. Ollitrault, Phys. Lett. B 641 (2006) 260 [nucl-th/0607009].

[16] B. Alver, B. B. Back, M. D. Baker, M. Ballintijn, D. S. Barton, R. R. Betts, R. Bindel and W. Busza et al., Phys. Rev. C 77 (2008) 014906 [arXiv:0711.3724 [nucl-ex]].

[17] J. -Y. Ollitrault, A. M. Poskanzer and S. A. Voloshin, Phys. Rev. C 80 (2009) 014904 [arXiv:0904.2315 [nucl-ex]].

[18] B. Alver and G. Roland, Phys. Rev. C 81 (2010) 054905 [Erratum-ibid. C 82 (2010) 039903] [arXiv:1003.0194 [nucl-th]].

[19] Z. Qiu and U. W. Heinz, Phys. Rev. C 84 (2011) 024911 [arXiv:1104.0650 [nucl-th]].

[20] S. Voloshin and Y. Zhang, Z. Phys. C 70, 665 (1996) [hep-ph/9407282]. 
[21] A. M. Poskanzer and S. A. Voloshin, Phys. Rev. C 58, 1671 (1998) [nucl-ex/9805001].

[22] B. Abelev et al. [STAR Collaboration], Phys. Rev. C 75 (2007) 054906 [nucl-ex/0701010].

[23] S. S. Adler et al. [PHENIX Collaboration], Phys. Rev. Lett. 91 (2003) 182301 [nucl-ex/0305013].

[24] A. Adare et al. [PHENIX Collaboration], Phys. Rev. Lett. 98 (2007) 162301 [nucl-ex/0608033].

[25] S. A. Voloshin, Nucl. Phys. A 715, 379 (2003) [nuclex/0210014].

[26] D. Molnar and S. A. Voloshin, Phys. Rev. Lett. 91 (2003) 092301 [nucl-th/0302014].

[27] V. Greco, C. M. Ko and P. Levai, Phys. Rev. C 68 (2003) 034904 [nucl-th/0305024].

[28] R. J. Fries, B. Muller, C. Nonaka and S. A. Bass, Phys. Rev. C 68 (2003) 044902 [nucl-th/0306027].

[29] R. C. Hwa and C. B. Yang, Phys. Rev. C 70 (2004) 024904 [hep-ph/0312271].

[30] M. Gyulassy and M. Plumer, Phys. Lett. B 243, 432 (1990).

[31] X. N. Wang and M. Gyulassy, Phys. Rev. Lett. 68, 1480 (1992).

[32] R. J. M. Snellings, A. M. Poskanzer and S. A. Voloshin, arXiv:nucl-ex/9904003.

[33] X. N. Wang, Phys. Rev. C 63, 054902 (2001).

[34] L. Adamczyk et al. [STAR Collaboration], Phys. Rev. Lett. 110 (2013) 142301 [arXiv:1301.2347 [nuclex]].

[35] L. Adamczyk et al. [STAR Collaboration], Phys. Rev. C 88 (2013) 014902 [arXiv:1301.2348 [nucl-ex]].

[36] B. B. Abelev et al. [ALICE Collaboration], arXiv:1405.4632 [nucl-ex].

[37] H. Song and U. W. Heinz, Phys. Lett. B 658 (2008) 279 [arXiv:0709.0742 [nucl-th]].

[38] H. Song and U. W. Heinz, Phys. Rev. C 77 (2008) 064901 [arXiv:0712.3715 [nucl-th]].

[39] H. Song and U. W. Heinz, Phys. Rev. C 78 (2008) 024902 [arXiv:0805.1756 [nucl-th]].

[40] H. Song, S. A. Bass, U. Heinz, T. Hirano and C. Shen, Phys. Rev. Lett. 106 (2011) 192301 [Erratum- ibid. 109 (2012) 139904] [arXiv:1011.2783 [nucl-th]].

[41] H. Song, S. A. Bass, U. Heinz, T. Hirano and C. Shen, Phys. Rev. C 83 (2011) 054910 [Erratum-ibid. C 86 (2012) 059903] [arXiv:1101.4638 [nucl-th]].

[42] H. Song, S. Bass and U. W. Heinz, arXiv:1311.0157 [nucl-th].

[43] C. Shen, U. Heinz, P. Huovinen and H. Song, Phys. Rev. C 84 (2011) 044903 [arXiv:1105.3226 [nucl-th]].

[44] B. Abelev et al. [ALICE Collaboration], Phys. Rev. C 88 (2013) 4, 044910 [arXiv:1303.0737 [hep-ex]].

[45] C. Adler et al. [STAR Collaboration], Phys. Rev. C 66 (2002) 034904 [nucl-ex/0206001].

[46] S. Voloshin, A. M. Poskanzer, and R. Snellings, in Relativistic Heavy Ion Physics, Landolt-Bornstein Vol. 1 (Springer-Verlag, Berlin, 2010), pp. 5-54.

[47] A. Adare et al. [PHENIX Collaboration], Phys. Rev. C 85 (2012) 064914 [arXiv:1203.2644 [nucl-ex]].

[48] A. Shor, Phys. Rev. Lett. 54 (1985) 1122.

[49] B. B. Abelev et al. [ALICE Collaboration], Phys. Lett. B 736 (2014) 196 [arXiv:1401.1250 [nucl-ex]].

[50] V. Khachatryan et al. [CMS Collaboration], JHEP 1009 (2010) 091 [arXiv:1009.4122 [hep-ex]].

[51] S. Chatrchyan et al. [CMS Collaboration], Phys. Lett. B 718 (2013) 795 [arXiv:1210.5482 [nucl-ex]].

[52] B. Abelev et al. [ALICE Collaboration], Phys. Lett. B 719 (2013) 29 [arXiv:1212.2001 [nucl-ex]].

[53] G. Aad et al. [ATLAS Collaboration], Phys. Rev. Lett. 110 (2013) 18, 182302 [arXiv:1212.5198 [hepex]].

[54] B. B. Abelev et al. [ALICE Collaboration], Phys. Lett. B 726 (2013) 164 [arXiv:1307.3237 [nucl-ex]].

[55] B. B. Abelev et al. [ALICE Collaboration], Phys. Rev. C 90 (2014) 5, 054901 [arXiv:1406.2474 [nuclex]].

[56] G. Aad et al. [ATLAS Collaboration], Phys. Lett. B 725 (2013) 60 [arXiv:1303.2084 [hep-ex]].

[57] G. Aad et al. [ATLAS Collaboration], arXiv:1409.1792 [hep-ex].

[58] A. Adare et al. [PHENIX Collaboration], arXiv:1404.7461 [nucl-ex]. 